\title{
Le nettoyage ethnique en ex-Yougoslavie : le cas de la Krajina de Knin
}

Ethnic cleansing in former Yougoslavia: the case of Krajina of Knin

Die ethnische Säuberung in Ex-Jugoslawien : Der Fall der Krajina von Knin

\section{Nicolas Lejeau}

\section{OpenEdition}

\section{Journals}

Édition électronique

URL : http://journals.openedition.org/rge/597

ISSN : 2108-6478

Éditeur

Association des géographes de l'Est

Édition imprimée

Date de publication : 1 janvier 2005

Pagination : 45-53

ISSN : 0035-3213

Référence électronique

Nicolas Lejeau, «Le nettoyage ethnique en ex-Yougoslavie : le cas de la Krajina de Knin », Revue Géographique de l'Est [En ligne], vol. 45 / 1 | 2005, mis en ligne le 04 juin 2009, consulté le 07 septembre 2020. URL : http://journals.openedition.org/rge/597

Ce document a été généré automatiquement le 7 septembre 2020

Tous droits réservés 


\section{Le nettoyage ethnique en ex- Yougoslavie : le cas de la Krajina de Knin}

Ethnic cleansing in former Yougoslavia: the case of Krajina of Knin

Die ethnische Säuberung in Ex-Jugoslawien : Der Fall der Krajina von Knin

\section{Nicolas Lejeau}

\section{NOTE DE L'ÉDITEUR}

Article reçu le 17 mai 2005, accepté le 3 juin 2005

1 Les politiques d'homogénéisation ethnique pratiquées en ex-Yougoslavie durant les années 1990 eurent un impact important aussi bien sur le plan démographique que territorial, social ou culturel. Dans cette optique, la Krajina de Knin offre un terrain d'étude aussi intéressant que symbolique. En effet, jusqu'à l'indépendance de la Croatie en 1991, cette région de l'arrière-pays dalmate était très majoritairement peuplée de Serbes, qui s'insurgèrent et érigèrent ce territoire en région autonome autoproclamée, avant de chasser l'essentiel de la population croate. Après quatre années de sécession, les forces croates reprirent militairement la Krajina durant l'été 1995. Cette reconquête généra une seconde vague d'expulsion massive qui visa cette fois les Serbes.

On reviendra donc dans cet article sur la localisation, l'histoire (ancienne marche militaire) et la spécificité ethnoculturelle de ce territoire avant d'examiner l'évolution récente de sa structure ethnique et de s'interroger sur les diverses conséquences de ces deux politiques successives. 


\section{Un territoire flou et original}

Le Krajina de Knin est un territoire difficile à délimiter - un territoire "flou » suivant l'expression de C. Rolland-May - et dont la délimitation diffère par conséquent selon les auteurs et les sources. Le terme même de Krajina ne désigne pas une région spécifique, c'est un terme générique qui regroupe une catégorie particulière d'espaces singularisés par leur rôle militaire. En effet, le terme Krajina (krajine au pluriel) vient du slave « kraj » qui signifie " fin » ou " limite » ${ }^{1}$, et désigne les anciennes Vojna Krajina ou Marches militaires de l'empire austro-hongrois (Militärgrenze en allemand). Il s'agit des régions frontalières que les Habsbourg mirent en place à partir de la seconde moitié du $\mathrm{XVI}^{\mathrm{e}}$ siècle tout le long de ce qui était alors la frontière austro-turque. Cette ceinture frontalière, à l'instar de systèmes défensifs plus anciens comme les marches carolingiennes, devait protéger leur empire des attaques turques. Notons que la petite région de Knin ne faisait pas initialement partie de cette «Vojna Krajina». En effet, avec l'arrière-pays dalmate, elle formait la région frontalière de la République de SaintMarc (Venise) jusqu'à son intégration à l'empire autrichien en $1815^{2}$. Ces régions furent peuplées de différentes nationalités, dont les Serbes qui étaient particulièrement nombreux dans les anciens territoires vénitiens, ce que nous appelonsKrajina de Knin.

4 Au sens le plus large, certains auteurs englobent sous le terme de Krajina de Knin toutes les anciennes régions de Croatie où les Serbes étaient majoritaires : cette vision large regroupait donc la Lika, la région de Knin, le Kordun, la Banija (au nord de Bihać) mais aussi certains secteurs de la Slavonie. Selon d'autres représentations, la Krajina regroupait les espaces précédemment cités mais sans la Slavonie. Enfin, pour d'autres auteurs, dans son sens le plus restreint, la Krajina de Knin n'inclurait que les environs immédiats de cette petite ville dalmate. Face à ces configurations quelque peu divergentes, on étudiera sous ce terme, la région de Knin à laquelle on associera la Banija, le Kordun et la Lika ${ }^{3}$ (figure 1), soit l'ensemble des territoires adossés à la frontière ouest de la Bosnie-Herzégovine en excluant surtout la Slavonie, plus proche à tous points de vue de la Voïvodine (Bataković, 1994). 
Figure 1 :Les Krajina de Croatie.

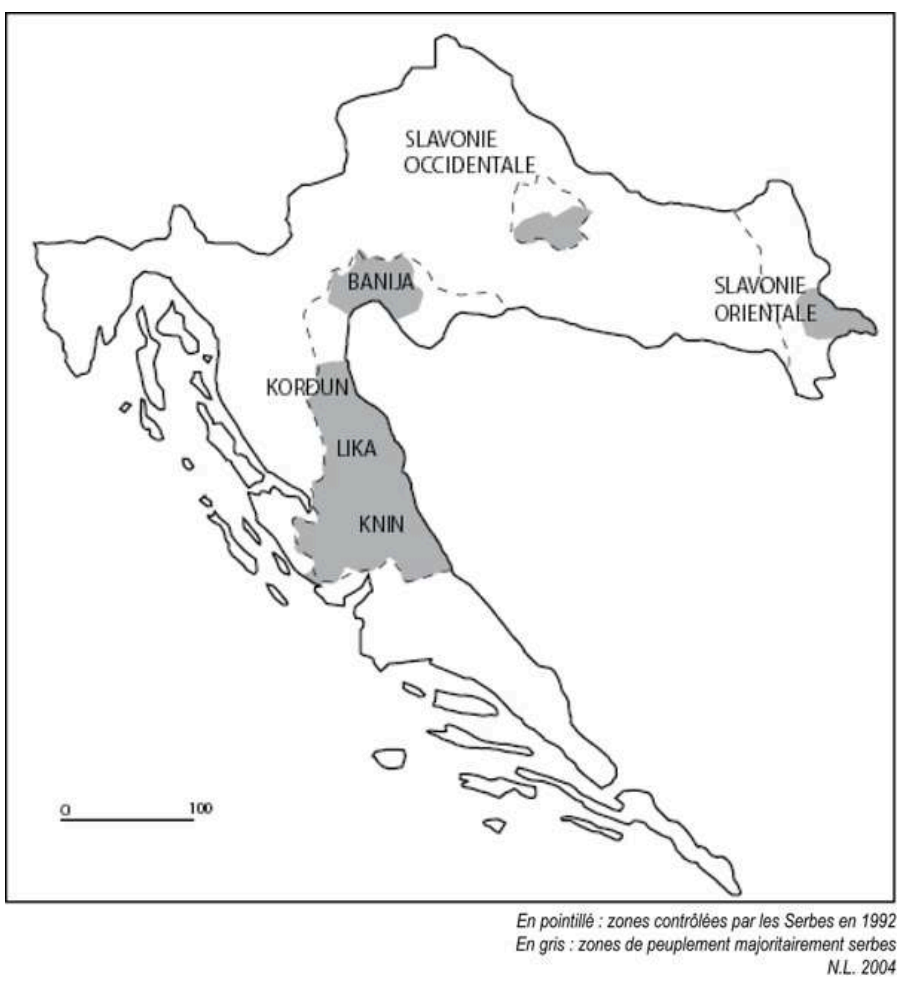

5 Le territoire de l'aire d'étude prend la forme, du sud au nord, d'une longue et étroite bande de territoire, le long de l'actuelle frontière croato-bosniaque. S'étendant de l'arrière-pays dalmate, terres calcaires et rocailleuses entrecoupées de poljés caractérisent la Lika et la région de Knin - semblables à beaucoup d'autres arrière-pays méditerranéens, et qui sont les terres les plus pauvres et les moins densément peuplées de toute la Croatie -, jusqu'à la Banija et au Kordun, régions collinaires situées au sud de la plaine fertile de la Save, à une soixantaine de kilomètres de Zagreb.

La particularité de cet espace résulte cependant surtout de sa spécificité ethnique. Depuis plusieurs siècles, dans une Croatie où ils constituent une minorité, les Serbes étaient, dans cette Krajina, en position majoritaire. La présence de ces Serbes sur ces terres est ancienne et trouve ses origines, pour l'essentiel, dans la domination habsbourgeoise. Les Habsbourg peuplèrent en effet ces terres peu peuplées de nombreux paysans-soldats serbes qui fuyaient le joug turc. Ces réfugiés étaient nommés «Uskoks», c'est-à-dire littéralement les "gens qui sautaient par dessus la frontière $»^{4}$. Ainsi, la région de Knin, partie la plus ancienne du défunt État médiéval croate, accueillait une population majoritairement serbe. Après la Grande Migration des Serbes du Kosovo en 1690, bon nombre de nouveaux émigrés serbes s'installèrent dans ces régions considérées comme sûres. Les habitants de ces confins militaires, appelés Granicari, du croate Granica : frontière (Nouzille, 1991, p. 67), avaient un statut particulier. Ils n'étaient pas soumis aux astreintes féodales, mais ils pratiquaient le service militaire en contrepartie de l'usufruit des terres attribuées. En d'autres termes, ils cultivaient la terre qui leur avait été donnée et en même temps devaient prendre les armes en cas de guerre. En 1630, l'empereur Ferdinand II dota ces Marches de conditions particulières de liberté. Il y instaura une administration confiée à des officiers généralement serbes: les Krajisnici. Ces Krajine constituaient donc des 
provinces autonomes qui dépendaient directement de l'empereur et non de la Croatie, ce que les insurgés de 1991 ne manquèrent pas de rappeler... Les Habsbourg y confirmèrent également la liberté d'exercice du culte orthodoxe, ce qui renforça l'identité de la population serbe. L'empire autrichien disposait ainsi de troupes prêtes à défendre sa frontière méridionale et dont l'entretien ne lui coûtait presque rien. Ce système efficace, semblable à celui des Cosaques de Russie, perdura jusqu'à la fin du XIX ${ }^{e}$ siècle (Nouzille, 1991). Conservé durant le court intermède napoléonien des Provinces Illyriennes (1809-1814), il ne fut définitivement aboli qu'en 1881, c'est-à-dire trois ans après l'occupation de la Bosnie-Herzégovine par l'Autriche-Hongrie (qui l'annexera définitivement en 1908). En effet, à cette date, l'Autriche n'avait plus, dans cette région, de frontières à défendre. Les Confins, majoritairement serbes furent alors réintégrés à la Croatie.

7 Cette histoire atypique, qui n'est pas sans évoquer celle de la Voïvodine, explique la structure ethnique particulière de cette région. La Krajina était une mosaïque ethnique et religieuse où coexistaient des modes de vie et des traditions fortement individualisées. Il a toujours existé de profondes différences entre les traditions de ces frontaliers libres serbes et celles du reste de la population croate. La société croate, catholique, féodale, hiérarchisée et caractérisée par l'émergence de classes moyennes et intellectuelles, faisait face à la société serbe des Confins, égalitaire, plus axée sur les activités agricoles et militaires. Les Serbes y ont donc conservé un particularisme culturel et un certain goût pour la carrière militaire. En outre, notamment dans la petite région de Knin, ils développaient un réel sentiment nationaliste, tradition qui a perduré jusqu'à l'éclatement de la Yougoslavie.

\section{La prédominance serbe de 1991}

8 Tous les recensements de populations confirment que les Serbes restèrent durablement majoritaires sur ces portions de Croatie jusqu'à 1995. Au milieu du XIXe siècle, d'après le recensement de 1857, les Serbes constituaient plus de la moitié de la population de l'ensemble des anciennes marches militaires (Garde, 2001, p. 67). D'après le recensement de 1931, les Serbes représentaient en moyenne 55,7\% dans la totalité des districts de la Krajina que nous appelons ici de Knin (Djuric, 1999, p. 94). En 1991, à la veille des combats, la Krajina comptait approximativement 270000 habitants. Les Serbes y composaient près de 70 à $75 \%$ de la population tandis que les Croates étaient près de 25 à $30 \%$, soit 90000 personnes selon Michel Foucher (1996, p. 193-195). La figure 2 retranscrit cette hégémonie serbe. 
Figure 2 :L'hégémonie serbe de 1991.

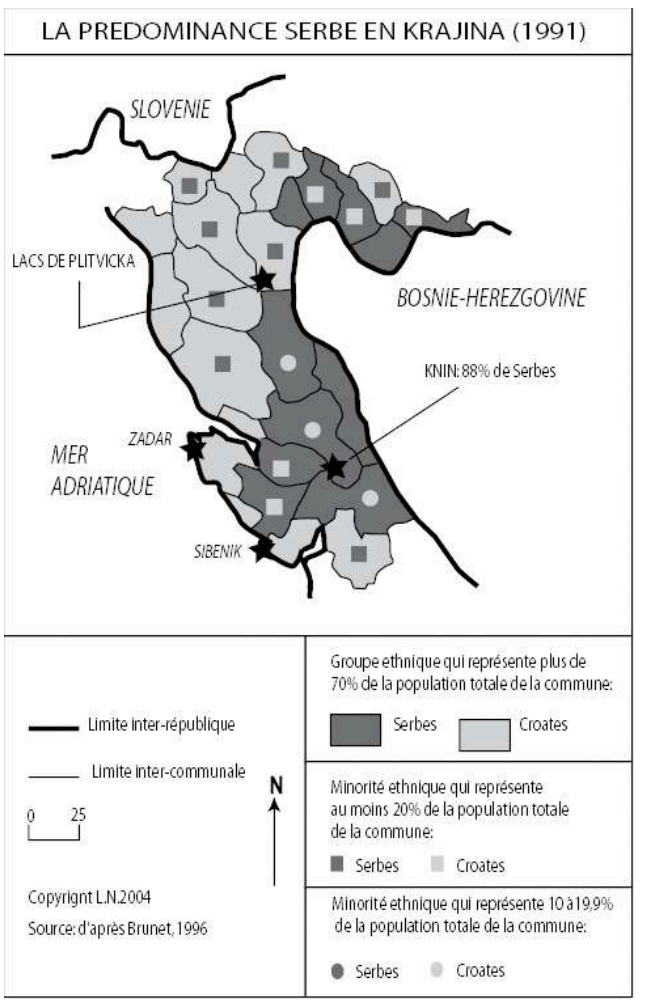

9 Les Serbes constituaient la majorité absolue dans onze communes (opstina en serbe, opcina en croate - espace vaste qui regroupe une ville centre et plusieurs villages à peu près équivalant à un canton français), qui correspondaient à $12 \%$ du territoire de la République de Croatie. Toutefois, il n'y résidait que 194000 des 580000 Serbes alors recensés en Croatie. La grande majorité des Serbes vivaient en effet en ville, et notamment à Zagreb (Derens et Samary, 2000, p. 179). Les Serbes vivaient donc aussi bien en ville qu'à la campagne tandis que les Croates de Krajina étaient principalement établis en zone urbaine. Cette répartition ethnique se retrouvait à l'échelle des communes. Les Serbes représentaient plus de $80 \%$ de la population dans les communes de Gračac, de Titova Korenica et de Donji Lapac. Ils étaient environ $70 \%$ dans les communes de la Lika situées entre Knin et le littoral adriatique (Obrovac et Benkovac). Dans le Kordun et dans la Banija, les communes de Vojnić, de Vrgin Most, de Glina, de Kostajnica et de Dvor na Uni comptaient également, selon le recensement de 1991, près de $70 \%$ de Serbes. Cette structure se retrouvait surtout à l'échelle communale, mais également à l'échelle urbaine. Ainsi, en 1991, la ville de Knin était presque intégralement peuplée de Serbes puisqu'il y formaient $88,6 \%$ de la population, soit 12 000 de ses 14000 habitants (Djuric, 1999, p. 100). On retrouvait des données similaires dans les communes voisines: plus de $80 \%$ de Serbes à Benkovac ou à Gračac (www.amb-croatie.fr).

\section{La guerre de Krajina : les différentes vagues d'homogénéisation}

10 Le problème de la Krajina est un parfait résumé du problème serbo-croate sensible à l'échelle de toute l'ex-Yougoslavie. Il y est particulièrement amplifié et exacerbé. 
Intimement liée à la question minoritaire, cette crise explosa au début des années 1990 . Revenons en détail sur ces événements emblématiques.

En mai 1990, eurent lieu les élections présidentielles croates au terme desquelles Franjo Tudjman, chef du parti nationaliste $\mathrm{HDZ}^{5}$, fut élu président. Le 25 juillet, le nouveau Parlement croate, le Sabor, dominé par ces mêmes nationalistes (en lieu et place des communistes), s'empressa de voter une série d'amendements remettant en cause le statut constitutionnel du peuple serbe. Zagreb supprima tout usage officiel de l'alphabet cyrillique et prit une série de mesures pour «croatiser» la langue. À la différence de la Croatie communiste qui, tout en reconnaissant diverses " nationalités ", était définie comme le foyer de deux peuples constitutifs, les Croates et les Serbes, la nouvelle Constitution adoptée définissait la république croate comme "l'État national de tous les Croates": "la République de Croatie se constitue en État national du peuple croate qui est aussi l'État de ceux qui, tout en appartenant à d'autres nationalités et à des minorités, sont ses citoyens : Serbes, Hongrois, Bosniaques, Italiens... ». De ce fait, la Croatie ne reconnaissait à ces derniers que la "citoyenneté ». Ainsi, les 580000 Serbes soit 12,2 \% de la population de la Croatie en 1991 (Derens et Samary, 2000, p. 358) gardaient leurs droits civiques mais perdaient le statut de peuple constitutif : ils avaient ainsi les mêmes droits que les Hongrois ( $0,5 \%$ de la population de Croatie)! Or, les Serbes refusaient d'être considérés comme une minorité, un groupe de seconde zone. Fondamentalement, les principes de la législation croate ont changé la nature du problème: c'est le respect des droits individuels des Serbes dans les régions où ils ne vivaient plus en groupe compact qui était remis en cause. Chez les Croates a toujours existé un complexe "du droit historique", au nom duquel ils affirmaient qu'il n'existait en Croatie qu'un seul peuple politique. Le problème récurrent de la reconnaissance d'une identité nationale serbe était donc ouvert. En outre, la nouvelle Croatie se dota d'un nouveau drapeau qui reprenait le damier rouge et blanc des blasons croates du Moyen Âge. Aux yeux des Serbes, il s'agissait plus d'une reprise de l'emblème de l'État croate des Oustachis, seul le nombre de cases du damier ayant été modifié. Ces "provocations» du nouveau pouvoir croate instauraient un climat d'inquiétude et semblaient justifier la propagande nationaliste et anti-croate de Belgrade. Beaucoup de Serbes crurent en effet à la résurrection de l'État oustachi, aidés en cela par les commentaires des médias serbes. Le président $\mathrm{F}$. Tudjman fut traité de "fasciste", son gouvernement désigné comme "Oustachi», les Croates furent présentés comme un "peuple génocidaire ", en référence aux exactions oustachis de la Seconde Guerre mondiale. Slobodan Milošević s'appuya sur les peurs des Serbes, leur sentiment d'insécurité croissant, tout en agitant le thème de la Grande Serbie pour les inciter au séparatisme sur la base d'une rébellion armée.

Durant l'année 1990, le psychiatre Jovan Raskovic et quelques intellectuels nationalistes créèrent un parti nationaliste, le Parti Démocratique Serbe (SDS de ses initiales serbocroates), qui prétendait représenter les Serbes de Croatie. Les nationalistes serbes prirent prétexte des mesures votées par le Sabor pour franchir le pas. Le jour même, plusieurs dizaines de milliers de Serbes rassemblés dans la bourgade au nom évocateur de Srb proclamèrent la souveraineté des communes contrôlées par le SDS. Dans les régions de Croatie dominées par les Serbes, les meetings se multiplièrent, reprenant en masse ces accusations. Le 17 août 1990, ces treize communes proclamèrent leur autonomie sous le nom de "Région autonome serbe", placée sous la direction d'un Conseil national serbe. Au départ centrée sur Knin, cette région autonome ne tarda pas 
à s'étendre en direction de la Lika et du Kordun. Au même moment, Milošević parvint à évincer Raskovic au profit du plus radical maire de Knin, Milan Babic. En août, le Conseil national serbe décida d'organiser un référendum d'autonomie. Les autorités croates jugèrent cette action illégale et tentèrent de pénétrer dans les communes qui s'étaient soustraites à son autorité. Des incidents armés éclatèrent aussitôt. À Knin, Milan Babic décréta l'état d'alerte et le chef, serbe, de la police de Knin, Milan Martic, se saisit des réserves d'armes de la ville pour les distribuer à la population. En quelques heures, toutes les routes de la région se couvrirent de barricades. Les médias croates appelèrent cela «la révolution des rondins ». Cet événement marqua le début du soulèvement armé de la Krajina, l'armée yougoslave encourageant discrètement les insurgés. La télévision de Belgrade multipliait les reportages sur l'événement, en montrant pour la première fois sans aucune censure, les insignes tchetniks et les autres manifestations du folklore nationaliste serbe. La Krajina de Knin était en révolte ouverte contre Zagreb.

13 Après le référendum où, sans surprise, $99,2 \%$ des électeurs serbes se prononcèrent en faveur de l'autonomie, la situation paraissait gelée (Castellan, 1991, p. 559). Belgrade décida d'approvisionner en armes les rebelles. Le 11 novembre 1990, la police croate interpella un groupe de convoyeurs d'armes près de la localité de Dvor na Uni. Le chef de ce groupe n'était autre qu'Arkan, qui retrouva la liberté peu de temps après en échange d'une forte rançon. Au début de 1991, la situation empira et le 28 février, les autorités de la "Région autonome » proclamaient la sécession d'avec la Croatie et leur volonté de s'unir avec la Serbie, le Monténégro et les Serbes de Bosnie. La région sécessionniste prit le nom de "République de Serbie - Région autonome serbe de Krajina " (RASK). La référence au mot "Krajina » servait de thème unificateur et identitaire. La Croatie fraîchement indépendante est ainsi contestée sur son sol par une entité autoproclamée non reconnue au niveau international (un pseudo-État).

Après que le Sabor eut abrogé les lois yougoslaves sur l'ensemble du territoire croate, les sécessionnistes serbes annexèrent, fin mars, le Parc naturel des lacs de Plitvice, en Lika, célèbre pour ses lacs et ses cascades. La police croate lança une forte opération de commando le dimanche de Pâques : un policier croate et deux Serbes furent tués. La guerre de Krajina comptait ses premiers morts. D'autres incidents suivirent et catalysèrent la psychose et l'engrenage de la peur et de la haine. Des barrages se multiplièrent aux abords des villages insurgés. Au plus fort de la crise, le 25 mars 1991, Milošević et Tudjman se rencontrèrent à Karadjordjevo pour se partager la dépouille de l'ex-Yougoslavie: les peurs des Serbes de Krajina, pions sur ce complexe échiquier, étaient instrumentalisées! La suite des événements le confirma puisque, le 25 juin 1991, la Croatie se proclama indépendante.

15 Dès lors, les combats se généralisèrent. À la fin du mois d'août, 38 communes, soit un tiers des 102 communes que comptait la Croatie, étaient en état d'insurrection et échappaient au contrôle des forces croates. La Krajina insurgée s'étendait alors sur $13913 \mathrm{~km}^{2}$. Les forces serbes y appliquèrent une drastique politique de «nettoyage ethnique ». L'armée fédérale, qui occupait jusque-là les territoires, laissait faire les milices serbes. Parmi ces bandes faisant régner la terreur, on peut citer la Garde des volontaires serbes d'Arkan. En un laps de temps très court, la Krajina fut littéralement expurgée de la grande majorité de sa population croate. Au cessez-le-feu de janvier 1992, on estimait que près de 80000 Croates, soit environ $90 \%$ de leur nombre total, avaient été chassés de leurs foyers. Les villes étaient toutes devenus quasi- 
exclusivement serbes (www.unhcr.com). Toutefois, si la population croate fut extrêmement touchée, les civils serbes furent également touchés par la guerre. En représailles, des irréguliers Croates incendièrent de nombreux villages à majorité serbe, terrorisant des Serbes locaux. Ainsi, même si cet aspect de la guerre en Krajina est méconnu, on estime que 80000 Serbes ont quitté volontairement la Krajina entre 1991 et 1995 (RSF, 1993, p. 213 et www.unhcr.com). Ces départs s'expliquaient bien plus par le chômage croissant et la crise économique grave que connaissait la région insurgée que par les exactions.

16 À l'époque de la RASK, on considérait que la Krajina comptait environ 220000 habitants répartis entre $88 \%$ de Serbes, $7 \%$ de Croates et $5 \%$ d'autres (beaucoup de réfugiés bosniaques; Weibel, 2002, p.582). L'homogénéisation violente offrait un résultat concret pour les nationalistes serbes.

Le cessez-le-feu signé à Sarajevo le 2 janvier 1992 gela momentanément les fronts, plus du quart du territoire croate échappait à l'autorité de Zagreb. Les Serbes durent accepter le déploiement des Casques bleus de l'ONU. En février, le leader serbe de la RASK, Milan Babic, qui s'opposait à ce déploiement - qu'avait approuvé Milošević - fut démis de ces fonctions et remplacé par le chef de la police de Knin, Milan Martic. Sous la direction de ce dernier, le pseudo-État allait survivre trois années. Durant la guerre de Bosnie, la Krajina ne prit pas directement part aux combats, mais elle s'impliqua dans de juteuses affaires de contrebande alors que la population survivait de plus en plus difficilement.

La Krajina, devenue une nouvelle "terre sainte serbe ", jouxtait la Republika Srpska de Bosnie. Elle formait donc un ensemble de territoires en continuité avec la Mère-Patrie serbe, toujours dans cette optique pan-nationale de Grande Serbie. Ces insurgés serbes se voulaient les plus farouches défenseurs des valeurs nationales, et cela du fait de leurs traditions (leurs ancêtres n'avaient-ils pas défendu le monde chrétien contre les Musulmans ?). Foyer d'indépendance et de nationalisme, la Krajina était devenue le cœur d'une nouvelle Serbie. La ville de Knin étant, de fait, la capitale de cette nouvelle Serbie qui se voulait « plus serbe que serbe».

19 Les offensives croates modifièrent véritablement la situation à l'été 1995. Les forces de Zagreb, bénéficiant du soutien américain, lancèrent la surprenante opération « Tempête » (Bljesak en serbo-croate) qui fit tomber en seulement trois jours la place forte de la rébellion (Knin), puis la Lika et le Kordun. Les forces serbes n'opposèrent presque aucune résistance, et les dirigeants de Knin, à commencer par M. Martic, s'enfuirent durant les combats. Les Serbes évacuèrent manu militari la population serbe de la Krajina. La reconquête a donc provoqué la fuite de 180000 Serbes qui payèrent leur soutien envers la politique de Belgrade. Ce chiffre représente l'immense majorité de la population serbe établie dans la région depuis des siècles (Lutard, 1998, p. 114). Ces départs formèrent donc une deuxième vague de nettoyage ethnique. Ils se sont principalement effectués en direction de la Slavonie orientale, encore sous contrôle serbe, où se sont entassés près de 50000 réfugiés, mais également en République serbe de Bosnie et en Serbie (Voïvodine). Le régime serbe tenta aussi d'installer plusieurs dizaines de milliers de ces réfugiés au Kosovo, mais en vain. En 1995, la Krajina de Knin ne comptait plus que 10000 à 12000 Serbes, selon les données des autorités croates de l'époque (www.ined.fr). 


\section{La nouvelle structure ethnique de la Krajina}

la Krajina est totalement inversée par rapport à la situation d'avant-guerre. Aujourd'hui, les Serbes forment une minorité en Krajina puisqu'ils ne représentent plus qu'environ $20 \%$ de la population locale (alors qu'en 1991, ils en représentaient près de trois-quarts).

21 L'analyse précise de cette modification radicale du peuplement est compliquée par le fait que le pavage administratif croate a été modifié en 1995. Les modifications du maillage territorial croates datent des semaines qui suivirent la fin du conflit. En général, trois à six anciennes communes ont été refondues pour en former une nouvelle, ce changement a donc donné naissance à des entités de taille conséquente (figure 3). Désormais, la Croatie se subdivise en 21 départements, les zupanije ${ }^{6}$. Si le but initial est de favoriser les études administratives (lors des recensements par exemple), il est incontestable que ce découpage "horizontal " (l'ancienne Krajina s'orientait de façon nord-sud) avait un objectif plus politique, plus nationaliste : affaiblir les positions serbes d'un point de vue statistique et électoral. Des remaniements administratifs de ce type (réunion de plusieurs communes où une ethnie est dominante avec des communes où une autre ethnie est majoritaire) permet en effet de rendre artificiellement la première ethnie partout minoritaire. Le remodelage des communes croates n'est pas un processus unique, on retrouve le même processus en Slovaquie où les communes à population hongroise (sud du pays) n'ont pas été supprimées mais réunies de force au sein de régions perpendiculaires à l'axe principal du pays, créant ainsi un nouveau maillage administratif où les Hongrois sont partout minoritaires. En Croatie, le remaniement a porté ses fruits et les données du recensement de 2001 confirment très nettement le recul démographique de la population serbe dans l'ancienne Krajina. 
Figure 3 : Nouveaux départements et anciennes communes.

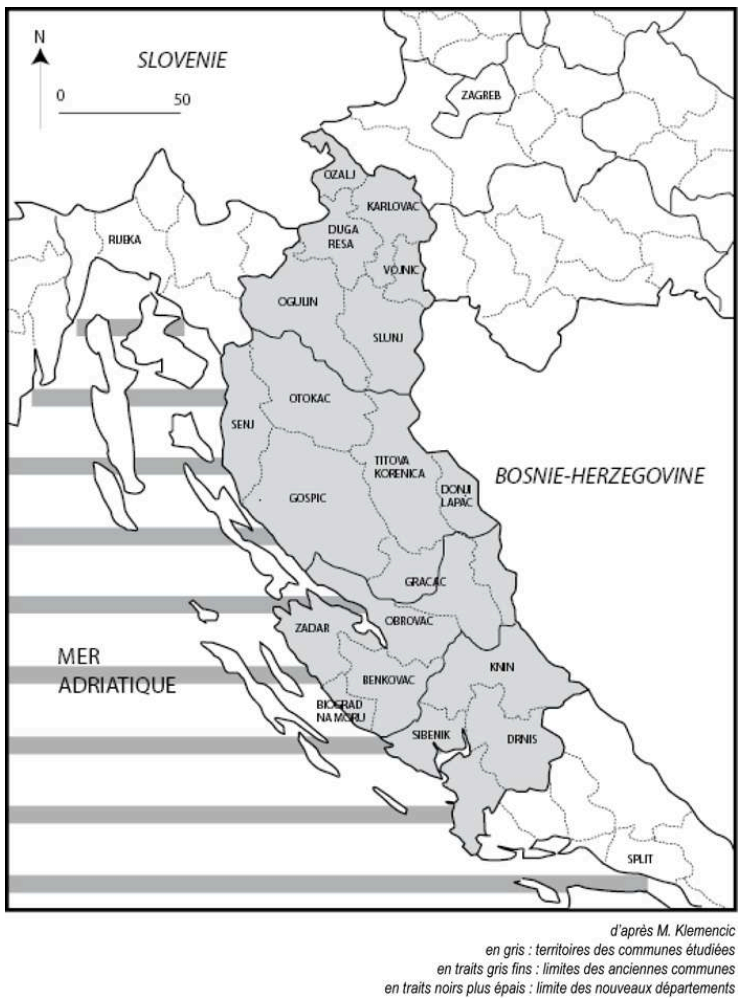

Tableau 1 : Évolution de la population serbe dans les communes croates de 1991 à 2001.

\begin{tabular}{|c|c|c|c|c|c|c|c|}
\hline $\begin{array}{l}\text { Communes } \\
\text { actuelles }\end{array}$ & $\begin{array}{c}\text { Population } \\
\text { totale en } \\
2001\end{array}$ & $\begin{array}{c}\% \text { de } \\
\text { Serbes } \\
\text { en } \\
2001\end{array}$ & $\begin{array}{c}\text { Équivalences } \\
\text { (communes de 1991) }\end{array}$ & $\begin{array}{c}\text { Population } \\
\text { en } 1991\end{array}$ & $\begin{array}{c}\% \text { de } \\
\text { Serbes } \\
\text { en } \\
1991\end{array}$ & $\begin{array}{c}\text { Population } \\
\text { en } 1991 \\
\text { (maillage de } \\
\text { 1991) }\end{array}$ & $\begin{array}{c}\text { \% moyen } \\
\text { de Serbes } \\
\text { (maillage } \\
\text { de 1991) }\end{array}$ \\
\hline $\begin{array}{l}\text { ŠIBENIK- } \\
\text { KNIN }\end{array}$ & 112891 & 9 & $\begin{array}{l}\text { DRNIŠ } \\
\text { KNIN } \\
\text { ŠIBENIK }\end{array}$ & $\begin{array}{l}24169 \\
42954 \\
85002\end{array}$ & $\begin{array}{l}21,3 \\
88,6 \\
10,5\end{array}$ & 152125 & 39,77 \\
\hline ZADAR & 162045 & 3,53 & $\begin{array}{l}\text { BENKOVAC } \\
\text { BIOGRAD NA MORU } \\
\text { DONJI LAPAC } \\
\text { GRAČAC } \\
\text { OBROVAC } \\
\text { ZADAR }\end{array}$ & $\begin{array}{rl}33 & 378 \\
17 & 661 \\
4 & 027 \\
5 & 217 \\
11 & 557 \\
136572\end{array}$ & $\begin{array}{r}57,4 \\
3,7 \\
97,4 \\
82,3 \\
65,9 \\
10,5\end{array}$ & 208412 & 37,52 \\
\hline
\end{tabular}




\begin{tabular}{|c|c|c|c|c|c|c|c|}
\hline LIKA-SENJ & 53677 & 11,5 & $\begin{array}{l}\text { DONJI LAPAC } \\
\text { GRAČAC } \\
\text { GOSPIĆ } \\
\text { OTOČAC } \\
\text { TITOVA KORENICA } \\
\text { SENJ }\end{array}$ & $\begin{array}{r}4027 \\
5217 \\
29049 \\
24992 \\
11393 \\
9205\end{array}$ & $\begin{array}{r}97,4 \\
82,3 \\
31,1 \\
32,2 \\
75,8 \\
2,3\end{array}$ & 83883 & 38,06 \\
\hline KARLOVAC & 141787 & 11 & $\begin{array}{l}\text { DUGA RESA } \\
\text { KARLOVAC } \\
\text { OGULIN } \\
\text { OZALJ } \\
\text { SLUNJ } \\
\text { VOJNIĆ }\end{array}$ & $\begin{array}{r}30485 \\
81319 \\
29095 \\
14787 \\
18962 \\
8236\end{array}$ & $\begin{array}{r}6,6 \\
26,6 \\
35,3 \\
1,0 \\
29,8 \\
90,6\end{array}$ & 182884 & 31,26 \\
\hline
\end{tabular}

Les anciennes communes de Donji Lapac et Gračac ont été, approximativement, coupées en deux entre les deux nouveaux départements de Zadar et de Lika-Senj. Pour faciliter le calcul, nous sommes partis sur la base que la moitié du pourcentage de Serbes (donc 40,115\% pour Gračac) vivaient dans chaque entité divisée. En outre, nous avons volontairement exclu les îles de ce calcul, afin de n'avoir que des communes continentales et donner plus de pertinence à ce raisonnement.

Source: Premiers résultats du recensement de 1991, Bulletin statistique n 1934, Belgrade, office fédéral de statistiques, 1992.

Une première observation générale est la diminution de la population entre les deux recensements. La guerre, en plus des morts qu'elle a causé, a poussé bon nombre de personnes (dont des effectifs conséquents de Serbes) à quitter la Croatie. C'est ainsi qu'une majorité de Serbes a fui le pays pour se réfugier en Serbie, au Monténégro ou en Bosnie voisine. Fort logiquement, la proportion de Serbes a diminué partout, phénomène accentué par le remaniement administratif. Prenons l'exemple du nouveau département de Šibenik-Knin, qui regroupe trois communes (Drniš, Knin, Šibenik). Il compte actuellement $9 \%$ de Serbes, soit 4,5 fois moins qu'en 1991. Autrement dit, 4 habitants sur 10 de cette commune auraient été serbes, chose qui serait passée aux yeux des responsables croates comme une aberration. Les figures 3 et 4 , combinées au tableau, illustrent ce remaniement voulu. 
Figure 4 :La nouvelle prééminence croate.

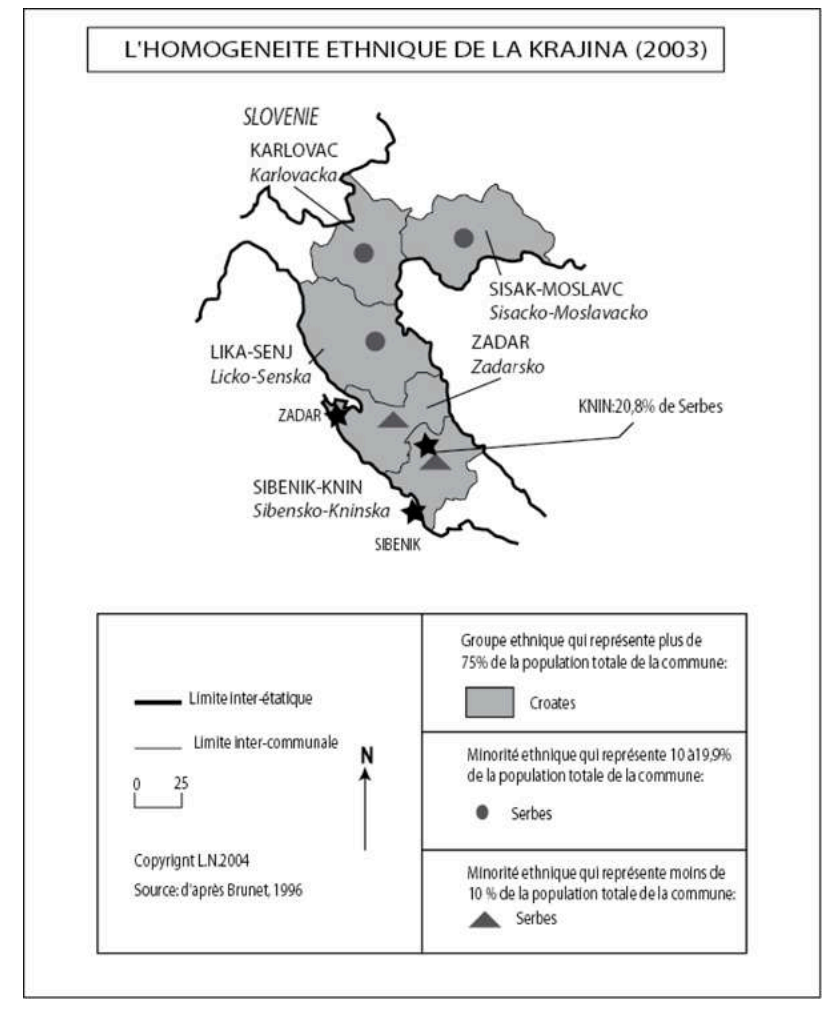

Dans le département de Šibenik-Knin, sur les 112891 habitants, 88,5\% sont des Croates (soit 99838 personnes). On ne trouve plus que 9,1\% de Serbes, soit près de 10300 ressortissants : c'est le groupe non croate le plus nombreux (au total, il y a 11263 non Croates). Si on rentre dans le détail, on s'aperçoit que le recul serbe est très important au niveau des villes. Ainsi à Knin (14 000 habitants), on trouvait 88 \% de Serbes en 1991. Au recensement de 2001, la ville comptait 15190 habitants dont 76,5\% de Croates (11 613 personnes) et $20,8 \%$ de Serbes (soit près de 3200 personnes). La même répartition s'observe dans les départements voisins. Ainsi, dans celle de Lika-Senj, les 53677 habitants se répartissent en $86 \%$ de Croates (46 245 personnes) et seulement $11,5 \%$ de Serbes (6 199 Serbes). On peut multiplier ces exemples : on trouve $84,3 \%$ de Croates (119 490 personnes) et $11 \%$ de Serbes (près de 16500 ressortissants) dans le département de Karlovac et sensiblement les mêmes chiffres dans celui de SisakMoslavina (82,1\% de Croates et $11,7 \%$ de Serbes, tous ces chiffres sont issus de www.dzs.hr). La figure 4 retranscrit cette nouvelle prééminence croate.

Toutefois, il ne faut pas généraliser ce recul car la situation diffère selon les espaces. Si on s'intéresse aux villages ruraux assez reculés, dans l'arrière-pays dalmate, on s'aperçoit que le recul serbe dans les zones rurales profondes n'est pas toujours aussi marqué. Beaucoup de Serbes sont restés. Ainsi, dans le cas du village d'Ervenik, sur les 988 habitants, on trouve encore 939 Serbes, soit $95 \%$ de la population du village, et seulement 31 Croates! Le même constat peut être fait à Biskupija: sur les 1669 habitants, 1290 sont serbes ( $77 \%$ de la population) et 377 Croates (20\%). Comment analyser ce phénomène? Il est certain que le recul serbe n'a pas eu la même ampleur entre les zones urbaines, plus parfaitement vidées de leur population Serbe, que dans les zones rurales. Dans les zones plus reculées, la population serbe qui est restée sur place est une population pauvre dépourvue de toute attache en Serbie. Ces Serbes sont 
bien souvent les descendants des paysans-soldats. Ce sont généralement des nationalistes convaincus, ils ont un tempérament fier et n'envisagent aucunement de quitter cette terre qu'ils considèrent comme la leur. Il ne faut donc pas généraliser cela car il ne faut pas omettre que les villes ont été plus sévèrement «nettoyées » que les campagnes. Ces grandes tendances sont modulables, ainsi, dans l'ancienne commune de Glina, la population a baissé de $60 \%$ entre les recensements de 1991 et 2002, mais la perte est de $35 \%$ dans la ville chef-lieu et de $72 \%$ ailleurs. Pourtant, il y avait $70 \%$ de Serbes dans la ville et $57 \%$ à la campagne (www.dzs.hr). La variation 1991-2002 dépend de l'intensité des combats, des destructions, des variations locales du "nettoyage » et aussi du nombre des retours, souvent plus nombreux dans les villes parce que c'est là que l'effort de reconstruction a été le plus poussé.

Cela s'explique également par la très faible proportion de retours des réfugiés serbes. On ne notait jusqu'à l'aube des années 2000, presque aucun retour serbe en Krajina car le parti nationaliste de Tudjman, le HDZ, veillait à empêcher par tous les moyens le retour des réfugiés et ainsi maintenir la « croatisation » du pays. Depuis le décès de ce dernier à la fin de l'année 2000, et surtout depuis la démocratisation du pays, on note une augmentation des retours de Serbes puisqu'on en recense environ 10000 à 20000 par an en moyenne (www.vlada.hr). Mais tout cela reste fragile car la minorité serbe craint toujours d'éventuelles représailles ou discriminations. Ainsi, en juin 2000, on ne comptait que 16500 retours de Serbes en Krajina. En outre, ces retours concernaient essentiellement une population âgée, surtout rurale, ce qui renforce le clivage ville/ campagne précédemment évoqué. Les recherches récentes soulignent que la plupart de ces retours sont temporaires. Les Serbes reviennent pour vendre leurs biens avant de repartir, généralement en Serbie.

La situation de la minorité croate chassée depuis 1991 n'est guère plus enviable. Son retour s'est très longtemps avéré difficile du fait de l'ampleur des destructions, du marasme économique de l'arrière-pays alors que, en Croatie comme dans d'autres secteurs du bassin méditerranéen, les tropismes sont littoraux. Toutefois, les retours croates sont plus conséquents. Leur situation psychologique et politique est évidemment nettement meilleure, ainsi environ 100000 réfugiés croates de Croatie mais également de Bosnie-Herzégovine se sont réinstallés depuis la fin du conflit (www.vlada.hr). La naturalisation de nombreux Croates de Bosnie-Herzégovine définitivement installés en Croatie a accentué la « croatisation » de nombreuses régions dont la Krajina de Knin.

Ces retours n'empêchent cependant pas le vieillissement et le déclin démographique de cette région de Dalmatie. L'importance des destructions, qu'elles soient matérielles (terrains minés, multiplication des friches...), ou moins visibles (désorganisation de l'appareil productif...), les obstacles psychologiques (rancœur entre communautés, entre voisins...) participent de la mise en périphérie de cet espace dans le territoire croate.

\section{Conclusion}

Ainsi, la Krajina de Knin apparaît comme un exemple éloquent de mutation du peuplement liée à des politiques d'homogénéisation ethnique. Elle a été l'un des premiers enjeux territoriaux de la guerre de Croatie et fut le premier symbole de la violence interethnique, avant même la Bosnie-Herzégovine. L'expulsion de la grande 
majorité de la population croate par les insurgés serbes dès 1991, puis quatre ans plus tard, la fuite de la population serbe ont complètement bouleversé le peuplement de cette région. Aujourd'hui, les Serbes y sont très minoritaires alors qu'ils formaient le socle de la population de cet espace avant-guerre. Si la violence interethnique n'est pas le seul facteur explicatif des mutations du peuplement (exode rural et chômage y participent aussi), la Krajina de Knin offre tout de même un exemple éloquent de mutation du peuplement consécutive à des violences politiques.

\section{BIBLIOGRAPHIE}

BATAKOVIĆ D. (1994). - Yougoslavie. Nations, religions, idéologies, Lausanne, L’Âge d'Homme, 355 p.

CASTELLAN G. (1991). - Histoire des Balkans XIV-XXe siècle, Paris, Fayard, 643 p.

Derens J.-A., SAMARY C. (2000). - Les conflits en Yougoslavie de A à Z, Paris, Éditions de l'Atelier, $427 \mathrm{p}$.

DJURIC I. (1999). - Glossaire de l'espace yougoslave, Paris, L'Esprit des Péninsules, 302 p.

FOUCHER M., dir., (1996). - Fragments d'Europe, Paris, Fayard, 327 p.

GARDE P. (2001). - Fin de siècle dans les Balkans, Paris, Odile Jacob, 264 p.

GARDE P. (1999). - Vie et mort de la Yougoslavie, Paris, Fayard, 480 p.

GRMEK M., GJIDARA M., SIMAC N. (1993). - Le Nettoyage ethnique, documents historiques sur une idéologie serbe, Paris, Fayard, 340 p.

Le Nouvel Observateur, Reporters Sans Frontières (1993). - Le livre noir de l'ex-Yougoslavie :

purification ethnique et crimes de guerres, Paris, Arléa, 485 p.

LUTARD C. (1998). - Géopolitique de la Serbie-Monténégro, Bruxelles, Complexe, 143 p.

NOUZILLE J. (1991). - Histoire de frontières, l'Autriche et l'Empire ottoman, Paris, Berg international, $263 \mathrm{p}$.

WeIBEL E. (2002). - Histoire et géopolitique des Balkans de 1800 à nos jours, Paris, Ellipses, Collection « l'Orient politique », $641 \mathrm{p}$.

\section{Webographie}

www.afebalk.org (site de l'association française des Études sur les Balkans).

www.amb-croatie.fr (site de l'ambassade de Croatie en France).

www.dzs.hr (site croate sur le recensement 2001 en Croatie).

www.ined.fr (site offrant de nombreuses données démographiques sur l'ex-Yougoslavie).

www.unhcr.com (site du Haut Commissariat aux réfugiés).

www.vlada.hr (site croate sur le retour des réfugiés en Croatie). 


\section{NOTES}

1. Krajina a notamment donné le nom « Ukraine ».

2. Il ne faut pas omettre l'épisode des provinces illyriennes qui commence en 1805 pour l'Istrie et la Dalmatie vénitiennes (Knin incluse) et en 1809 pour les territoires pris aux Habsbourgs, dont le reste de la Krajina étudiée ici.

3. La continuité du peuplement majoritairement serbe de la Krajina était interrompue par la commune majoritairement croate de Slunj (découpage de 1991, cf. figure 3).

4. Du verbe « uskaiti », littéralement sauter dedans.

5. Littéralement, il s'agit de la « communauté démocratique croate », parti principal du pays.

6. Certaines publications parlent de «comtés» reprenant la traduction «counties » que l'on trouve dans l'abondante littérature anglo-américaine. En effet, c'est un découpage d'échelle infra-régionale qui témoigne de la volonté centraliste du pouvoir.

\section{RÉSUMÉS}

La Krajina de Knin, en Croatie, est un parfait exemple de territoire ayant subi plusieurs nettoyages ethniques et ce dans un laps de temps assez court. Ancienne marche militaire des Habsbourg (Militärgrenze ou vojna krajina), cette région fut le théâtre d'un double nettoyage ethnique durant l'éclatement de l'ancienne Yougoslavie (1991-95). Lors de l'accès à l'indépendance de la Croatie, la Krajina de Knin était majoritairement peuplée de Serbes qui s'insurgèrent et en expulsèrent la population croate en 1991. Après quatre années d'affrontements, et d'indépendance autoproclamée, la Krajina fut reconquise par les forces croates durant l'été 1995. À cette occasion, la Krajina de Knin fut vidée de l'essentiel de sa population serbe. L'impact de ces événements est toujours sensible aussi bien par la dépopulation engendrée, que dans les paysages, l'économie ou les mentalités.

The Krajina of Knin, in Croatia, is a good example of a territory that suffered various ethnic cleansing within a short period. An old military frontier of the Habsburg monarchy (Militärgrenze in German or vojna Krajina in Serbo-croatian) the territory of the Knin's Krajina was the scene of a double ethnic cleansing when the former Yougoslavia collapsed. Indeed when Croatia became an independant State in June 1991 this region was populated by a majority of ethnic Serbians. Those Serbians rose up against the authority of Zagreb and erected an independant state of krajina from which they expelled all ethnic Croatians people. After four years of secession the Krajina of Knin was reconquered by Croatians forces during the summer of 1995. During this reconquest the Serb population fled eastward to serbian Bosnia or Serbia. The impact of these violent events is still obvious : the region is now underpopulated, and merely purely with ethnic Croatians, it is marginalized from an economic point of view and the mentalities still prevent the return of refugees and any attempt of reconciliation.

Die Krajina von Knin ist ein perfektes Beispiel für ein Territorium, das mehrere ethnische Säuberungen erlitten hat, und dies in einem ziemlich kurzen Zeitraum. Als früheres militärisches Grenzland von Habsburg (vojna Krajina) wurde diese Region der Schauplatz einer doppelten ethnischen Säuberung während des Zusammenbruchs des ehemaligen Jugoslawien (1991-1995). Seit des Unabhängigkeit Kroatiens war die Krajina von Knin mehrheitlich von Serben bevölkert, 
die sich auflehnten und die kroatische Bevölkerung 1991 vertrieben. Nach vier Jahren der Konfrontation und der selbstproklamierten Unabhängigkeit wurde die Krajina durch die kroatischen Kräfte während des Sommers 1995 wieder erobert. Bei dieser Gelegenheit wurde die Krajina vom Grossteil seiner serbischen Bevölkerung geleert. Die Auswirkung dieser Ereignisse ist noch immer spürbar - sowohl durch die erzeugte Entvölkerung als auch in den Landschaften und Mentalitäten.

\section{INDEX}

Schlüsselwörter : Bevölkerungsverschiebung, Demographie, Ethnische Säuberung, Krajina, Kroatien

Keywords : Croatia, demography, ethnic cleansing, Krajina, population removal

Mots-clés : Croatie, démographie, déplacement de population, Krajina, nettoyage ethnique

\section{AUTEUR}

\section{NICOLAS LEJEAU}

CERPA, Université Nancy 2, BP 33-97, F-54015 Nancy Cedex, France 